\title{
Does whole-body cryotherapy improve vertical jump recovery following a high-intensity exercise bout?
}

This article was published in the following Dove Press journal:

Open Access Journal of Sports Medicine

24 February 2015

Number of times this article has been viewed

\author{
Amilton Vieira' \\ Martim Bottaro' \\ Joao B Ferreira-Junior ${ }^{1,4}$ \\ Carlos Vieira' \\ Vitor A Cleto' \\ Eduardo L Cadore ${ }^{2}$ \\ Herbert G Simões ${ }^{3}$ \\ Jake Do Carmo' \\ Lee $\mathrm{E}$ Brown ${ }^{5}$ \\ 'College of Physical Education, \\ University of Brasília, Brasília, \\ ${ }^{2}$ College of Physical Education, \\ Federal University of Rio Grande \\ do Sul (UFRGS), Porto Alegre, \\ ${ }^{3}$ College of Physical Education, \\ Catholic University of Brasília, \\ Brasília, ${ }^{4}$ Federal Institute of Triângulo \\ Mineiro, Paracatu, Brazil; ${ }^{5}$ Department \\ of Kinesiology, California State \\ University, Fullerton, CA, USA
}

\begin{abstract}
Whole-body cryotherapy (WBC) has been used as a recovery strategy following different sports activities. Thus, the aim of the study reported here was to examine the effect of WBC on vertical jump recovery following a high-intensity exercise (HIE) bout. Twelve trained men (mean \pm standard deviation age $=23.9 \pm 5.9$ years) were randomly exposed to two different conditions separated by 7 days: 1 ) WBC ( 3 minutes of $\mathrm{WBC}$ at $-110^{\circ} \mathrm{C}$ immediately after the HIE) and 2) control (CON; no WBC after the HIE). The HIE consisted of six sets of ten repetitions of knee extensions at $60^{\circ} \cdot \mathrm{s}^{-1}$ concentric and $180^{\circ} \cdot \mathrm{s}^{-1}$ eccentric on an isokinetic dynamometer. The vertical jump test was used to evaluate the influence of HIE on lower extremity muscular performance. The vertical jump was performed on a force platform before HIE (T1) and 30 minutes after (T2) the WBC and CON conditions. As a result of HIE, jump height, muscle power, and maximal velocity $\left(\mathrm{V}_{\max }\right)$ had significant decreases between $\mathrm{T} 1$ and $\mathrm{T} 2$, however no significance was found between the WBC and CON conditions. The results indicate that one session of WBC had no effect on vertical jump following an HIE compared with a CON condition. WBC may not improve muscle-function (dependent on stretch-shortening cycle) recovery in very short periods (ie, 30 minutes) following HIE.
\end{abstract}

Keywords: functional performance, muscular recovery, countermovement jump

\section{Introduction}

High-intensity exercise (HIE) performed during a training session or competition may temporarily impair human performance; this impairment may last minutes, hours, or several days following the exercise bout. ${ }^{1}$ Thus, several treatment strategies have been suggested to optimize muscle recovery. ${ }^{2}$ Among the most common approaches used to restore muscle function are nonsteroidal anti-inflammatory drugs, massage techniques, stretching, exercise, compression garments, ultrasound, electrical current, and cryotherapy. ${ }^{1,2}$ For decades cryotherapy has been used as a postexercise recovery strategy $\mathrm{y}^{3,4}$ and a recent meta-analysis concluded that cold-water immersion is an efficient strategy to reduce delayed-onset muscle soreness following different exercise bouts. ${ }^{5}$

Whole-body cryotherapy (WBC) was originally proposed for the treatment of rheumatic disorders in the 1970s. ${ }^{6}$ WBC consists of exposure to very cold air $\left(-110^{\circ} \mathrm{C}\right.$ to $\left.195^{\circ} \mathrm{C}\right)$ for $2-4$ minutes. ${ }^{7}$ The claims made about the benefit of WBC to human performance are based on positive effect on hematological profile ${ }^{8}$ and the antioxidant system, ${ }^{9}$ improved lipid profile (low-density lipoprotein decrease and highdensity lipoprotein increase), ${ }^{10}$ and increased immunity. ${ }^{9}$ These beneficial responses have reasoned the use of WBC as a recovery strategy following different sports activities. ${ }^{7}$
College of Physical Education, University of Brasilia, QD 107 LT 2/4 Apt-102C.

7|919-700 Brasília, Brazil

Tel +55 6l 82805500

$\mathrm{Fax}+556133072085$

Email amilton_educacao@hotmail.com 
Despite an increased number of studies on the effects of WBC on the human body few studies have investigated the effectiveness of WBC on muscle-performance recovery. ${ }^{11-13}$ Among these studies, Hausswirth et $\mathrm{al}^{13}$ compared WBC with passive recovery in hastening muscular recovery within the 48 hours after a running protocol designed to induce muscle damage. Results of this study suggested greater muscle strength after the WBC session (post-1 hour) compared with passive recovery. On the other hand, Costello et $\mathrm{al}^{14}$ showed that WBC is ineffective in alleviating muscle soreness or enhancing muscle-strength recovery following exercise when WBC treatment was performed 24 hours after an eccentric muscle-damage exercise protocol. Thus, the results regarding the potential effect of WBC on muscle recovery after HIE remain controversial.

Therefore, the purpose of this study was to compare the effect of WBC with control conditions on jump-performance recovery following an HIE session. Our hypothesis was that WBC exposure would improve muscle performance after exercise.

\section{Methods}

\section{Subjects}

Sample size was determined a priori using $\mathrm{G}^{*}$ Power (v 3.1.6; University of Trier, Trier, Germany) with the level of significance set at 0.05 and power at 0.80 . A pilot study was conducted with four subjects to find the magnitude of the difference between means of the $\mathrm{CON}$ and WBC conditions. Effect sizes of 0.49 and 0.61 for power output and jump height, respectively, were found. Based on these calculations twelve resistance-trained males (data shown as mean \pm standard deviation; age $23.9 \pm 5.9$ years, body mass $92.5 \pm 10.4 \mathrm{~kg}$, height $180.5 \pm 5.8 \mathrm{~cm}$, and body mass index $28.4 \pm 2.8 \mathrm{~kg} \cdot \mathrm{cm}^{-2}$ ) were recruited to participate in this study. To ensure comparable responses to WBC exposure, subjects with similar features, like training history and body mass index, were recruited. ${ }^{15}$ Subjects who had practiced noncompetitive resistance exercise for the purpose of muscle hypertrophy for at least 1 year, with no interruptions longer than 20 consecutive days and for at least 2 years before the study, were recruited. Their training routine included four to six split-training sessions per week, performing six to eight sets per muscle group, and six to twelve maximum repetitions per set with 60 to 120 seconds of rest interval between sets. They were informed of the purpose, procedures, possible discomforts, risks, and benefits of the study prior to signing written informed consent.

Subjects were determined healthy and fit for physical exercise based on their answers to the Physical Activity Readiness Questionnaire (PAR-Q). ${ }^{16}$ Also, based on FerreiraJunior et al, ${ }^{17}$ the following exclusion criteria were adopted: untreated arterial hypertension, cardiovascular or respiratory diseases, angina, peripheral artery occlusive disease, venous thrombosis, urinary tract diseases, severe anemia, allergy to cold, tumor diseases, viral and bacterial infections, Raynaud's syndrome, claustrophobia, or convulsions. During the testing schedule, the subjects were instructed to maintain their normal hydration and their normal diet practices.

This study was approved by the University of Brasilia Human Research Ethics Committee (Protocol: 71484/2012) and was performed in accordance with the Declaration of Helsinki ethical standards.

\section{Experimental design}

This study had a randomized, controlled, crossover design. The subjects were assigned, using a random-numbers generator (randomization.com), to start with either the WBC or CON condition. Seven days later the subject repeated the other treatment. Firstly, subjects visited the testing laboratory for anthropometric measurements and experiment procedure familiarization. Then, subjects performed the first exercise session and, 7 days after, the second session. Each exercise session consisted of muscle-function testing involving a first vertical jump test (T1), HIE followed by a rest period of 30 minutes, and then a second vertical jump

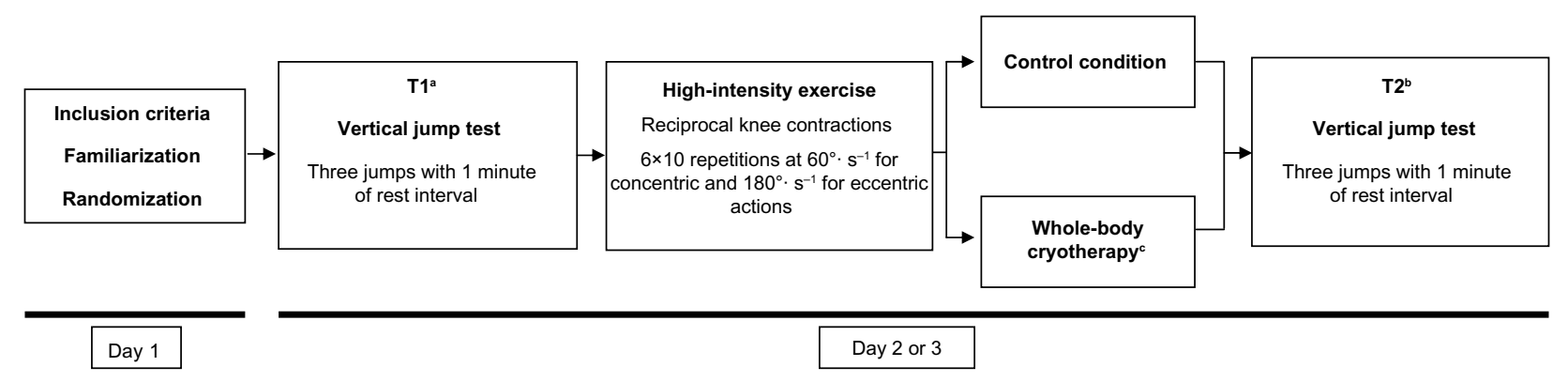

Figure I Experimental design.

Notes: aPre-exercise vertical jump test; ${ }^{b}$ postexercise vertical jump test; ${ }^{c} 3$ minutes of treatment at $-110^{\circ} \mathrm{C}$. 
test (T2) (Figure 1). The WBC exposure was administered immediately after the HIE for 3 minutes and the volunteers were allowed to sit and drink water ad libitum during the remaining 27 minutes between the $\mathrm{WBC}$ and $\mathrm{T} 2$ in a room with a temperature of between $21^{\circ} \mathrm{C}$ and $25^{\circ} \mathrm{C}$. For the CON session the subjects remained seated in the same room for 30 minutes before performing $\mathrm{T} 2$.

\section{Vertical jump test}

Two vertical jump tests (each vertical jump test comprised three jumps) were performed pre-HIE (baseline) and 30 minutes post-recovery from HIE. No warm-up or stretching exercises were allowed before the jump test. The jumps were measured on an AMTI force plate (model BP400600-HF-2000; Advanced Mechanical Technology, Inc., Watertown, MA, USA), sampling at $1000 \mathrm{~Hz}$, and stored on a computer. Data were processed using a routine written in MATLAB software (v R2008a7, The MathWorks Inc., Natick, MA, USA).

For the vertical jump, the subjects placed their hands on their hips, performing three countermovement jumps as high as possible. A self-determined range of motion was permitted and the rest period between each jump was 1 minute. ${ }^{18}$ Data obtained during vertical jumps were captured from manufactured software (AMTI Acquisition Software, v 4.2; Advanced Mechanical Technology, Inc.). Ground reaction force was calculated from math models described by Linthorne. ${ }^{19}$ After this, curves of acceleration, velocity, and displacement were extrapolated. The velocity curve was obtained by dividing the resultant ground reaction force by the body mass of the subjects, which produced the acceleration curve. The displacement was obtained by numerically integrating the velocity signal. Finally, the displacement curve was integrated to obtain the displacement of center of mass at each instant of movement; the greatest vertical displacement was considered jump height. Power was obtained as the product of ground reaction force and velocity, and maximal velocity $\left(\mathrm{V}_{\max }\right)$ was identified as the greatest value in the velocity curve.

\section{High-intensity exercise protocol}

After baseline jump-performance, subjects completed a reciprocal exercise bout consisting of 60 maximal concentric and eccentric actions of the right knee extensors. The knee extension exercise consisted of six sets of ten repetitions with 2 minutes rest between sets. The velocity was set at $60^{\circ} \cdot \mathrm{s}^{-1}$ for concentric and at $180^{\circ} \cdot \mathrm{s}^{-1}$ for eccentric actions. Each concentric action was followed by an eccentric contraction to knee-extensor muscles. A range of motion of $80^{\circ}$ flexion-extension (excursion between $10^{\circ}$ and $90^{\circ}$ relative to a $0^{\circ}$ at full knee extension) was used. All exercise was performed on a Biodex System 3 Isokinetic Dynamometer (Biodex Medical, Inc., Shirley, NY, USA). The calibration and positioning procedures followed manufacturer's recommendations. Subjects sat in the chair in a manner that allowed the rotational axis of the dynamometer to remain aligned with the lateral epicondyle of the femur during exercise sessions. The lever arm was adjusted and fixed $5 \mathrm{~cm}$ above the calcaneus. The chair and dynamometer adjustments for each subject were recorded to ensure that the position remained constant throughout the testing period. Verbal encouragement and visual feedback through the dynamometer computer monitor were provided to encourage subjects to reach their maximum exertion level. ${ }^{20}$

\section{Recovery modalities}

For the WBC condition, subjects stood in a head-out cryocabin based on gaseous nitrogen (Kryos Tecnologia, Brasília, Brazil) at $-110^{\circ} \mathrm{C}$ for 3 minutes. In order to protect body extremities, subjects wore bathing suits, gloves, socks, and shoes with thermic protection. They were instructed to move around the cryo-cabin and to flex and extend their elbows and fingers throughout the 3 minutes.

For the CON condition, subjects followed the same procedures as the WBC condition however the chamber's temperature was $21^{\circ} \mathrm{C}$.

\section{Skin-temperature measurement}

Skin temperature was measured immediately before (pre-cooling) and after (post-cooling) the application of WBC. The temperature was measured on the anterior midline of the thigh, midway between the proximal border of the patella and the inguinal crease. A 566 Infrared Thermometer (Fluke Corporation, Everett, WA, USA) was perpendicularly positioned and kept as close as possible to the skin without touching.

\section{Statistical analyses}

Descriptive statistics (means and standard deviations) were used to report jump height, power, and maximal velocity data. The Shapiro-Wilk test showed that all data were normally distributed. Two-way, repeated-measures analyses of variance [condition $(\mathrm{WBC}$ and $\mathrm{CON}) \times$ time $(\mathrm{T} 1$ and $\mathrm{T} 2)$ ] were used to analyze each variable. In the case of significant difference, Tukey's post-hoc test was performed.

SigmaPlot (v 11; Systat Software, Inc., San Jose, CA, USA) was used for statistical analysis, with an alpha level set 
at 0.05 . Complementarily, the effect size (ES) calculation was used to examine the magnitude of the treatment effect. Cohen's ranges of $0.2,0.5$, and 0.8 were used to define small, medium, and large $d$-values, respectively, obtained from differences between pretest and posttest scores divided by the pooled standard deviation. ${ }^{21}$

\section{Results}

There was no significant difference in initial skin temperature of $33.5^{\circ} \mathrm{C} \pm 0.7^{\circ} \mathrm{C}$ among participants in the experimental groups $(P>0.05)$. WBC exposure caused a decrease in skin temperature after 3 minutes of application $(P<0.001)$ : after WBC exposure the skin temperature was $18.3^{\circ} \mathrm{C} \pm 7.1^{\circ} \mathrm{C}$, while in the control group no significant change was observed $(P>0.05)$.

The effects of HIE on vertical jump parameters (height, power, and $\mathrm{V}_{\max }$ ) are presented in Figure 2. Jump height
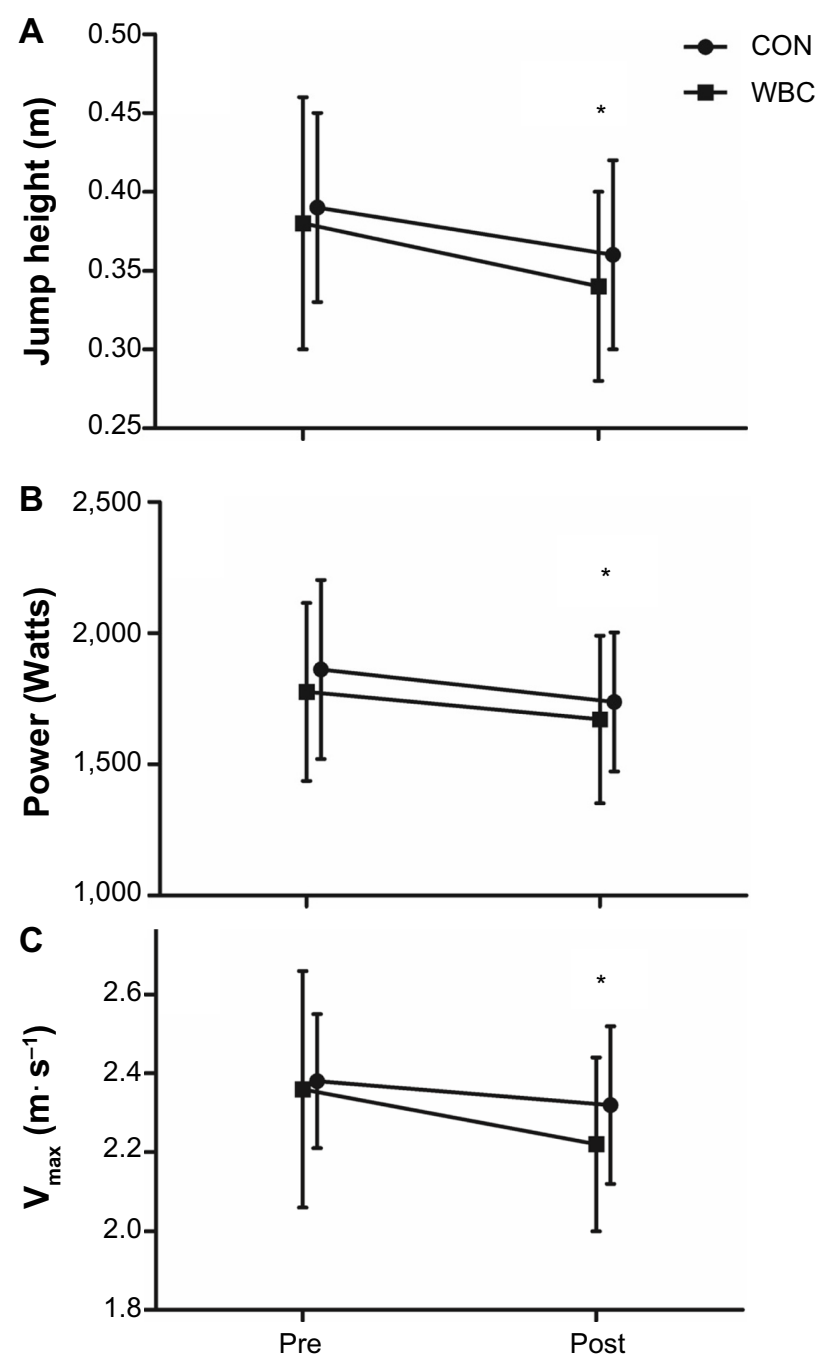

Figure 2 Vertical jump parameters before ("pre") high-intensity exercise and after ("post") whole-body cryotherapy (WBC) and control (CON) conditions. (A) Jump height; (B) muscle power; (C) maximal velocity $\left(\mathrm{V}_{\max }\right)$.

Note: *Lower than the pre value $(P<0.05)$. decreased significantly $(P<0.05)$ after the two conditions $(6.7 \%$ and $\mathrm{ES}=0.41$ for $\mathrm{CON} ; 10.1 \%$ and $\mathrm{ES}=0.47$ for WBC). However, no main effect for condition was found $(P=0.13)$. Power also decreased after both conditions $(P<0.05 ; 7.4 \%$ and $\mathrm{ES}=0.40$ for $\mathrm{CON} ; 9.7 \%$ and $\mathrm{ES}=0.44$ for WBC). No main effect for condition was found $(P=0.16)$. $\mathrm{V}_{\max }$ decreased after both conditions $(P<0.05 ; 2.8 \%$ and $\mathrm{ES}=0.34$ for $\mathrm{CON} ; 6.6 \%$ and $\mathrm{ES}=0.49$ for $\mathrm{WBC})$. No main effect for condition was found $(P=0.37)$.

\section{Discussion}

The main finding of the present study was that WBC had no effect on vertical jump (height, muscle power, and $\mathrm{V}_{\max }$ ) recovery 30 minutes after an intense training session composed of concentric and eccentric actions when compared with a control condition. This result is in opposition to our hypothesis that WBC would improve muscle recovery.

Our findings are relevant because short-term recovery is very important in sports activities that require at least two maximal performances with short rest intervals (ie, judo competitions; fast triathlons; half-time in basketball, soccer, and American football games). Therefore, the potential benefits of recovery strategies such as WBC need to be further explored, and, to the best of the authors' knowledge, this is the first study to have investigated the potential short-term benefits of WBC on stretch-shortening exercise performance following HIE.

Studies by Hausswirth et $\mathrm{al}^{13}$ and Fonda and Sarabon ${ }^{12}$ demonstrated that WBC accelerates recovery from exerciseinduced muscle damage to a greater extent than passive recovery. Fonda and Sarabon ${ }^{12}$ examined the effects of WBC on muscle-performance parameters during a 5-day recovery period. WBC was applied each day for 5 days during the recovery period and the exposures lasted 3 minutes at very low temperatures $\left(-140^{\circ} \mathrm{C}\right.$ to $\left.-195^{\circ} \mathrm{C}\right)$. Functional performance was evaluated by squat jump, counter movement jump, maximal isometric torque production, and rate of torque development. A significant improvement was observed in rate of torque development recovery after the WBC condition. Hausswirth et $\mathrm{al}^{13}$ reported that three sessions of WBC performed within 48 hours after a damaging running exercise bout accelerated recovery. These authors used a counter-balanced design to evaluate nine runners that performed 48 minutes of running on a treadmill, designed to induce muscle damage. Markers of muscle damage (kneeextensor strength, plasma creatine kinase, and perceived sensations [ie, pain, tiredness, well-being]) were recorded before, immediately after (post), 1 hour, 24 hours, and 48 
hours after exercise. WBC enhanced muscle strength 1 hour after exercise, whereas the same was not observed under the control condition. In addition, WBC also improved perceived sensations compared with the control condition. Moreover, no difference in plasma creatine kinase activity was observed between conditions.

In contrast, our results demonstrated that WBC performed immediately after high-intensity concentric-eccentric exercise has no effect on jump-performance recovery. Costello et $\mathrm{al}^{14}$ showed that WBC is ineffective in alleviating muscle soreness or enhancing muscle-force recovery. They observed that, after 100 eccentric knee extensions at a velocity of $90 \%$, WBC did not improve knee strength, power output, or muscle soreness at any time point when WBC was administered 24 hours after the eccentric exercise. These contradictory results are probably due to differences in cooling application time (ie, immediate application or 24 hours after exercise) and the different exercise type, duration, and intensity of the protocol used to induce muscle damage.

Previous studies have suggested that WBC exposure at $-110^{\circ} \mathrm{C}$ decreases muscle temperature for at least 60 minutes after exposure. ${ }^{14,22}$ This decrease promotes physiological responses such as decreased cell metabolism, posttraumatic capillary dysfunction, and enzymatic activity. ${ }^{4,23}$ Collectively, these responses may preserve musclefiber function and reduce exercise-induced muscle-damage symptoms. ${ }^{23}$ On the other hand, cold application may transitorily impair muscular performance. This transitory impairment may last from some minutes to 1 hour following the cold application. ${ }^{24,25}$ Thus, our results may be explained, at least in part, by the time the subsequent test was performed (30 minutes after WBC application). A possible benefit of WBC treatment could be masked by a deleterious effect of cold on muscular performance.

Studies investigating cooling effects using other cold modalities (ice or water immersion) also present contradictory results in force generation. ${ }^{26,27}$ Hopkins and Stencil ${ }^{26}$ investigated the effects of ice application on the ankle joint. H-reflex and concentric torque were measured before; immediately after; and 30, 60, and 90 minutes following the ice application. They found increased H-reflex and plantar flexion torque at all time points following ice application. In contrast, Dewhurst et $\mathrm{al}^{27}$ verified a decrease in isokinetic torque immediately after muscular cooling in a wide range of velocities ( 30 to $240^{\circ} / \mathrm{s}$ ). Recently, Vieira et $\mathrm{al}^{24}$ verified that cooling affects isometric and isokinetic torque production differently. They found an improvement in isometric torque, but a decrease in concentric isokinetic torque. Taken together, these results suggest that force development after muscular cooling is dependent on the cooled area (joint or muscle) and the muscle action performed (static or dynamic), all of which may influence the results.

The present results suggest that WBC is ineffective at accelerating jump-performance recovery 30 minutes after an intense training session composed of concentric and eccentric actions. However, caution should be taken when interpreting the present results because WBC was applied immediately after exercise and then the subsequent activity was performed 30 minutes after the exercise bout. Also, one possible limitation of the study was that just the right leg was trained and both legs were used for the jump test.

In this view, if exercise is performed soon after cold application, exercise performance may be decreased, particularly in high-intensity and explosive exercises. ${ }^{28}$ It should be recommended that the subject allow sufficient time for tissue to re-warm. In this regard, a warm-up before exercise performance could be useful. It is suggested that WBC would be ineffective in similar conditions, but one cannot exclude the possibility that WBC may generate positive effects when used for pain alleviation or when administered several times in the subsequent days following HIE to treat symptoms of muscle damage.

\section{Conclusion}

WBC had no effect on jump height, muscle power output, or $\mathrm{V}_{\max }$ recovery following a high-intensity concentric-eccentric exercise bout when compared with a control condition. From a practical standpoint, WBC does not improve muscle-function recovery in very short periods (ie, 30 minutes) following high-intensity concentric-eccentric exercise.

\section{Acknowledgment}

The authors would like to thank CAPES-Brazil and CNPqBrazil for their financial support.

\section{Disclosure}

The authors declare no conflicts of interest in this work.

\section{References}

1. Barnett A. Using recovery modalities between training sessions in elite athletes: does it help? Sports Med. 2006;36(9):781-796.

2. Cheung K, Hume P, Maxwell L. Delayed onset muscle soreness: treatment strategies and performance factors. Sports Med. 2003;33(2):145-164.

3. Banfi G, Lombardi G, Colombini A, Melegati G. Whole-body cryotherapy in athletes. Sports Med. 2010;40(6):509-517.

4. Knight KL. Cryotherapy in Sport Injury Management. Champaign, IL: Human Kinetics; 1995.

5. Leeder J, Gissane C, van Someren K, Gregson W, Howatson G. Cold water immersion and recovery from strenuous exercise: a meta-analysis. Brit J Sport Med. 2012;46(4):233-240. 
6. Rymaszewska J, Tulczynski A, Zagrobelny Z, Kiejna A, Hadrys T. Influence of whole body cryotherapy on depressive symptoms preliminary report. Acta Neuropsychiatr. 2003;15(3):122-128.

7. Selfe J, Alexander J, Costello JT, et al. The effect of three different $\left(-135^{\circ} \mathrm{C}\right)$ whole body cryotherapy exposure durations on elite rugby league players. PLoS One. 2014;9(1):e86420.

8. Lombardi G, Lanteri P, Porcelli S, et al. Hematological profile and martial status in rugby players during whole body cryostimulation. PLoS One. 2013;8(2):e55803.

9. Lubkowska A, Szygula Z, Klimek AJ, Torii M. Do sessions of cryostimulation have influence on white blood cell count, level of IL6 and total oxidative and antioxidative status in healthy men? Eur J Appl Physiol. 2010;109(1):67-72.

10. Lubkowska A, Banfi G, Dołegowska B, d’Eril GV, Łuczak J, Barassi A. Changes in lipid profile in response to three different protocols of wholebody cryostimulation treatments. Cryobiology. 2010;61(1):22-26.

11. Ziemann E, Olek A, Kujach S, Grzywacz T, et al. Five-day wholebody cryostimulation, blood inflammatory markers, and performance in high-ranking professional tennis players. J Ath Train. 2012;47(6): 664-672.

12. Fonda B, Sarabon N. Effects of whole-body cryotherapy on recovery after hamstring damaging exercise: a crossover study. Scand J Med Sci Sports. 2013;23(5):270-278.

13. Hausswirth C, Louis J, Bieuzen F, et al. Effects of whole-body cryotherapy vs far-infrared vs passive modalities on recovery from exercise-induced muscle damage in highly-trained runners. PLoS One. 2011;6(12):e27749.

14. Costello JT, Algar LA, Donnelly AE. Effects of whole-body cryotherapy $\left(-110^{\circ} \mathrm{C}\right)$ on proprioception and indices of muscle damage. Scand $J$ Med Sci Sports. 2012;22(2):190-198.

15. Cholewka A, Stanek A, Sieroń A, Drzazga Z. Thermography study of skin response due to whole-body cryotherapy. Skin Res Technol. 2012;18(2):180-187.

16. Thomas S, Reading J, Shephard RJ. Revision of the Physical Activity Readiness Questionnaire (PAR-Q). Can J Sport Sci. 1992;17(4): $338-345$.
17. Ferreira-Junior JB, Vieira CA, Soares SR, et al. Effects of a Single Whole Body Cryotherapy $\left(-110^{\circ} \mathrm{C}\right)$ Bout on Neuromuscular Performance of the Elbow Flexors during Isokinetic Exercise. Int J Sports Med. Epub September 25, 2014.

18. Markovic G, Dizdar D, Jukic I, Cardinale M. Reliability and factorial validity of squat and countermovement jump tests. $J$ Strength Cond Res. 2004;18(3):551-555.

19. Linthorne NP. Analysis of standing vertical jumps using a force platform. Am J Phys. 2001;69(11):1198-1204.

20. Brown LE, Whitehurst M, Bryant JR, Buchalter DN. Reliability of the Biodex System 2 isokinetic dynamometer concentric mode. Isokinet Exerc Sci. 1993;3(3):160-163.

21. Beck TW. The importance of a priori sample size estimation in strength and conditioning research. J Strength Cond Res. 2013;27(8): 2323-2337.

22. Costello JT, Culligan K, Selfe J, Donnelly AE. Muscle, skin and core temperature after $-110^{\circ} \mathrm{C}$ cold air and $8^{\circ} \mathrm{C}$ water treatment. PLoS One. 2012;7(11):e48190.

23. Guilhem G, Hug F, Couturier A, et al. Effects of air-pulsed cryotherapy on neuromuscular recovery subsequent to exercise-induced muscle damage. Am J Sports Med. 2013;41(8):1942-1951.

24. Vieira A, Oliveira AB, Costa JR, Herrera E, Salvini TF. Cold modalities with different thermodynamic properties have similar effects on muscular performance and activation. Int J Sports Med. 2013;34(10): 873-880.

25. Crowe MJ, O’Connor D, Rudd D. Cold water recovery reduces anaerobic performance. Int J Sports Med. 2007;28(12):994-998.

26. Hopkins JT, Stencil R. Ankle cryotherapy facilitates soleus function. J Orthop Sports Phys Ther. 2002;32(12):622-627.

27. Dewhurst S, Macaluso A, Gizzi L, Felici F, Farina D, De Vito G. Effects of altered muscle temperature on neuromuscular properties in young and older women. Eur J Appl Physiol. 2010;108(3):451-458.

28. Versey NG, Halson SL, Dawson BT. Water immersion recovery for athletes: effect on exercise performance and practical recommendations. Sports Med. 2013;43(11):1101-1130.
Open Access Journal of Sports Medicine

\section{Publish your work in this journal}

Open Access Journal of Sports Medicine is an international, peer-reviewed, open access journal publishing original research, reports, reviews and commentaries on all areas of sports medicine. The manuscript management system is completely online and includes a very quick and fair peer-review system.

\section{Dovepress}

Visit http://www.dovepress.com/testimonials.php to read real quotes from published authors. 